\title{
Accuracy of predictive magnification factor for preoperative templating in total hip arthroplasty
}

\begin{abstract}
The purpose of the present study was to evaluate the accuracy of predicted magnification factor (MF) for preoperative digital templating before THA in our institution.

A retrospective review of postoperative radiographs of individuals who received primary THA was conducted. The final sample included 531 patients with a mean age $66 \pm 12$ years, 221 of them were males (mean age $62 \pm 12$ years) and $310-$ females (mean age $68 \pm 11$ years). The biggest diameter of acetabular component in postoperative radiographs was measured twice at a one-month interval by observers. The measurements were made under MF of $0 \%$. The predictive MF of $15 \%$ is used at our institution. True MF was calculated as the measured size of acetabular component divided by the actual size multiplied by 100 . The accuracy of the predictive MF was calculated as the predictive MF divided by true MF multiplied by 100 .

The intra-observer ICC $0.93 \pm 0.01$ and inter-observer $0.95 \pm 0.02$ agreement was excellent. The mean true MF was $15.51 \%$ and accuracy of the predictive MF was $77.23 \%$. After the split by gender, the mean true MF was $15.84 \%$, accuracy of the predictive MF was $77.97 \%$ in males and $15.28 \%, 76.71 \%$ in females, respectively.

The predictive MF factor appeared to have too low accuracy for modern templating. We recommend assessing the true MF in hospitals where digital templating is routinely used for THA and consider using calibration markers in order to increase the accuracy of preoperative planning.
\end{abstract}

Keywords: hip arthroplasty, digital templating, PACS
Volume 10 Issue 2 - 2018

Povilas Masionis,' Justinas Jonušas, ${ }^{2}$ Martynas Karneckas, ' Valentinas Uvarovas,' Narunas Porvaneckas'

'Vilnius University Clinic of Rheumatology, Orthopaedic and Reconstructive Surgery, Centre of Orthopaedic and Traumatology, Republican Vilnius University Hospital, Lithuania ${ }^{2}$ Faculty of Medicine,Vilnius University, Lithuania

Correspondence: Povilas Masionis, Vilnius University Clinic of Rheumatology, Orthopaedic and Reconstructive Surgery, Centre of Orthopaedic and Traumatology, Republican Vilnius University Hospital, Šiltnamiu str. 29,Vilnius LT-04I30, Lithuania, Tel +37061822383, Email: povilasmasionis@yahoo.com

Received: January 29, 2018 | Published: March 14, 2018
Abbreviations: AP, anteroposterior; BMI, body mass index; $\mathrm{CM}$, calibration markers; DT, digital templating; ICC, intraclass correlation coefficient; MF, magnification factor; PACS, archiving and communication system; THA, total hip arthroplasty

\section{Introduction}

Total hip arthroplasty (THA) is one of the most common orthopedic procedures. It has proven to be very successful in reducing pain and restoring joint function..$^{1-4}$ Preoperative templating makes an essential part of the planning process before THA. It prepares an orthopedic surgeon for the procedure by reducing surgical time needed to measure the size of the implant. ${ }^{3,5}$ Furthermore, it minimizes the cost of the procedure related with inventory control and also helps to avoid postoperative complications. ${ }^{3,6-8}$

Preoperative templating could be classified into three types: 1) Traditional templating using acetate template on a hard copy of the anteroposterior (AP) radiograph of the pelvis; 2) Hybrid templating using conventional acetate templates on digital x-ray images; 3) Fully digital templating (DT).$^{7,9-12}$ Usually, an $\mathrm{x}$-ray image is magnified up to $20 \%$ because of the gap between bone and the film. This level of magnification may vary. ${ }^{13}$ Accordingly, acetate templates come already adjusted for $20 \%$ magnification. Such templating can produce errors during the preoperative planning stage, because the magnification factor (MF) is predicted theoretically and may vary depending on the gap between X-ray film and bone. ${ }^{9}$

After introduction of Picture Archiving and Communication System (PACS) in hospitals, digital templating is becoming more and more popular among orthopedic surgeons. ${ }^{10}$ DT software allows surgeons to change the magnification of $\mathrm{x}$-ray image and adapt it to the template. That helps to minimize the errors of templating produced by the magnification effect. In order to get the best results during the preoperative planning, accurate calculation of MF is needed. ${ }^{4,14,15}$

The purpose of the present study was to evaluate the accuracy of the predicted MF for preoperative digital templating for THA in our institution and to give recommendations to increase the accuracy of MF, accordingly.

\section{Materials and methods}

\section{Patients}

A retrospective review of postoperative radiographs of individuals who received primary THA of single hip was conducted. The data from a single institution was collected over one-year period (1 September, 2015 - 1 September, 2016) from the local register of hip arthroplasty. 632 patients who received primary THA for degenerative osteoarthritis, posttraumatic osteoarthritis, rheumatoid arthritis, aseptic necrosis of femoral head and hip joint dysplasia, were included. After analysis of the data, 101 patients were excluded (Table 1) and the final sample size included 531 cases with a mean age $66 \pm 12$ years: $221(41.6 \%)$ males, mean age $62 \pm 12$ years and $310(58.4 \%)$ females, mean age $68 \pm 11$ years. $388(73.1 \%)$ of the implants were cemented ones, $131(24.7 \%)$ mechanical and $12(2.2 \%)$ hybrid (11 cemented stem and 1 cemented cup)

\section{Methods}

Three independent orthopedic surgeons were invited to participate as observers during this study. Each of them had to measure the biggest diameter of acetabular component in postoperative radiographs after 
primary THA twice at one-month interval (Figure 1). Observers were provided as much time as needed for accurate evaluation of $\mathrm{x}$-ray photos. The measurements were made under MF of $0 \%$. The predictive MF of $15 \%$ is used by our institution during preoperative planning with the assumption that the distance between the $\mathrm{x}$-ray emitter and film is $110-115 \mathrm{~cm}$ and a patient is as close to the $\mathrm{x}$-ray film as possible. The actual size of acetabular component was taken from the local register of hip arthroplasty. True MF was calculated as the actual size of acetabular cup divided by the measured diameter of cup multiplied by 100 . The accuracy of predictive MF was calculated as the predictive MF divided by the true MF multiplied by $100.100 \%$ accuracy would indicate that the predictive MF and the true MF are equal, while lower value of accuracy indicates greater discrepancy between the true and predictive MF.

Table I Data analysis.

\begin{tabular}{ll}
\hline Initial sample size & $\mathbf{6 3 2}(\mathbf{1 0 0 \% )}$ \\
\hline Incorrect radiographs & $4(0.6 \%)$ \\
Absent radiographs & $16(2.5 \%)$ \\
Incorrect data in the register & $53(8.4 \%)$ \\
Absent acetabular cup size in the register & $5(0.8 \%)$ \\
Absolute exception (by z values) & $23(3.6 \%)$ \\
Final sample size & $\mathbf{5 3 1}(\mathbf{8 4 . 0 \% )}$ \\
\hline
\end{tabular}

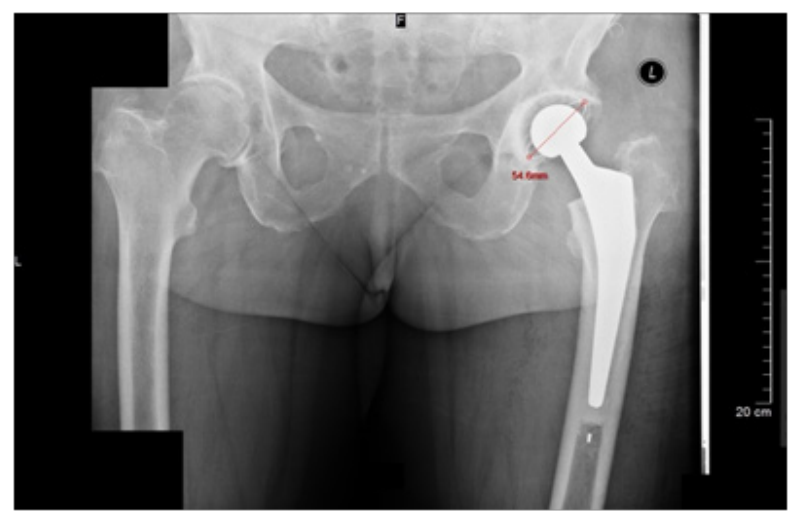

Figure I Measurement of the largest diameter of acetabular cup in postoperative radiograph after primary total hip arthroplasty.

\section{Statistical analysis}

The level of inter- and intra- observer reliability was determined by intraclass correlation coefficient (ICC), using its two-way mixed model. Reliability for absolute agreement was tested also. The following intervals were used to interpret the ICC values: less than 0.40 for poor agreement, $0.41-0.60$ indicates moderate agreement, $0.61-0.80$ indicates substantial agreement, and greater than 0.81 for almost perfect agreement. ${ }^{16}$ Statistical analysis was performed using SPSS v21.0 software. For additional calculations Microsoft Excel 2016 was used.

\section{Results}

The intra-observer ICC $0.93 \pm 0.01$ and inter-observer $0.95 \pm 0.02$ agreement was excellent. The mean true MF was $15.51 \pm 5.40 \%$ and accuracy of the predictive MF was $77.23 \pm 11.53 \%$. When true MF was divided into groups by gender, mean true MF was $15.84 \pm 5.17 \%$, accuracy of the predictive MF was $77.97 \pm 10.78$ in males and $15.28 \pm$ $5.54 \%, 76.71 \pm 12.03 \%$ in females, respectively. There was no statistically significant difference of true MF and accuracy between genders $(\mathrm{P}>.05)$.

\section{Discussion}

The most important finding of the present study was low accuracy of the predictive MF, which was only $77.23 \pm 11.53 \%$. Even though the mean true MF diverges from the predictive MF by $0.51 \%$ only $(15,51 \%$ vs $15 \%)$, there was a high variance around the mean. In our opinion, that was exactly the reason why there was such a low accuracy of the predictive MF.

Riddick et al. found that the accuracy of the preoperative MF (scaling ball was used) was $96 \%$ (range 89.6 - 99.9) in their institution. ${ }^{17}$ The author assumed that the accuracy would be even higher if the calculated true MF were considered equal across all patients as is the case for our institution. However, in our study the divergence between true $\mathrm{MF}$ and the predictive MF ranged from $2.17 \%$ to $24.58 \%$. We cannot agree with the above statement by Riddick et al. and recommend using the calibration markers (CM). Furthermore, the present study shows a simple method to evaluate accuracy of the predictive MF. In our opinion, it is essential to perform this self-assessment in all institutions where digital templating is used routinely, since accurate MF factor could help to reduce operating time, avoid intra and postoperative complications and finally, to improve patient safety.

The usage of external $\mathrm{CM}$ in order to predict $\mathrm{MF}$ is a technique proposed by Clarke et al. ${ }^{18}$ It has several limitations such as correct placement and shape of CM, discomfort for patients, etc. ${ }^{19,20}$ There were studies on positioning of spherical $\mathrm{CM}$ that proposed a formula to determine the vertical and horizontal position of $\mathrm{CM} .{ }^{4}$ The horizontal position of $\mathrm{CM}$ was shown to be less significant in comparison with the vertical position. ${ }^{15}$

Furthermore, it was found that CM should be placed at the height of greater trochanter without skin and bone overlap in order to obtain the greatest accuracy. ${ }^{21}$ Franken et al. ${ }^{22}$ compared two different ways to place CM: CM positioned laterally, at the height of the greater trochanter and $\mathrm{CM}$ positioned medially between the legs. Mean errors were $2.55 \%$ and $2.04 \%$, respectively. Another study compared the CM method with the distance measuring method. ${ }^{23}$ The authors concluded that measuring MF without $\mathrm{CM}$ is almost as accurate as measuring with $\mathrm{CM}$ (mean error with $\mathrm{CM} 2.6 \%$; without CM 2.8\%). $\mathrm{CM}$ was positioned laterally, near the greater trochanter. However, mean error of the predictive MF in our study was lower (only $0.51 \%$ ) in comparison with the abovementioned study, but high dispersion of the true MF around the predictive MF resulted in high inaccuracy. It seems the magnification error is unavoidable even when CM is used. Nevertheless, the magnification error is evidently lower when using $\mathrm{CM}$ and the use of the latter may improve the accuracy of the predictive MF. ${ }^{24}$

Amount of soft tissue between hip joint and x-ray detector affects magnification by increasing the gap between them, ${ }^{25}$ making it more difficult for a physician to locate a correct position to place the CM (e.g. at the height of greater trochanter) for more corpulent patients. ${ }^{25}$ Also, it becomes more difficult to keep a standardized distance between x-ray tube and detector for patients with increased BMI, which may lead to a potential risk of inaccuracy when predicting MF. Contrarily, a number of studies have shown no positive correlation between BMI and accuracy of MF. ${ }^{25,26}$ This appears to be highly consistent with our findings. We have found no significant difference in MF accuracy between male and female patients even though the elderly female patients are associated with larger hip circumference. ${ }^{27}$ 
The authors are aware of limitations of this study. Firstly, this is a retrospective study, without randomization. Secondly, there were too few observers. Further research investigation is needed to find out if MF could be significantly improved having introduced CM.

\section{Conclusion}

The predictive $\mathrm{MF}$ factor used in our institution $(\mathrm{MF}=5 \%)$ has appeared to have too low accuracy for modern templating. We recommend to assess true MF before every THA in hospitals where digital templating is routinely used and to consider using the calibration markers in order to increase the accuracy of preoperative planning.

\section{Acknowledgement}

None.

\section{Conflict of interest}

Authors declare there is no conflict of interest in publishing the article.

\section{References}

1. Khan F, Ahmad T, Condon F, et al. Review of quality of x-rays for templating for total hip arthroplasty. Acta Orthop Belg. 2015;81(1):726.

2. Inoue $\mathrm{D}$, Kabata $\mathrm{T}$, Maeda $\mathrm{T}$, et al. Usefulness of three-dimensional templating software to quantify the contact state between implant and femur in total hip arthroplasty. Eur J Orthop Surg Traumatol. 2015;25(8):1293-300.

3. Marcucci M, Indelli PF, Latella L, et al. A multimodal approach in total hip arthroplasty preoperative templating. Skeletal Radiol. 2013;42(9):1287-94.

4. Boese CK, Bredow J, Dargel J, et al. Calibration marker position in digital templating of total hip arthroplasty. $J$ Arthroplasty. 2016;31(4):883-7.

5. Ranjitkar S, Prakash D, Prakash R. Magnification error of digital $\mathrm{x}$ rays on the computer screen. Nepal Med Coll J. 2014;16(2-4):182-5.

6. Davila JA, Kransdorf MJ, Duffy GP. Surgical planning of total hip arthroplasty: accuracy of computer-assisted EndoMap software in predicting component size. Skeletal Radiol. 2006;35(6):390-3.

7. McAuley JP, Ridgeway SR. Preoperative planning to prevent dislocation of the hip. Orthop Clin North Am. 2001;32(4):579-586.

8. Pourmoghaddam A, Dettmer M, Freedhand AM, et al. A patientspecific predictive model increases preoperative templating accuracy in hip arthroplasty. J Arthroplasty. 2015;30(4):622-6.

9. Ries MD. CORR Insights ${ }^{\circledR}$ : acetate templating on digital images is more accurate than computer-based templating for total hip arthroplasty. Clin Orthop Relat Res. 2015;473(12):3760-1.

10. Hafez MA, Ragheb G, Hamed A, et al. Digital templating for THA: a simple computer-assisted application for complex hip arthritis cases. Biomed Tech (Berl). 2016;61(5):519-24.

11. Krishnamoorthy VP, Perumal R, Daniel AJ, et al. Accuracy of templating the acetabular cup size in total hip replacement using conventional acetate templates on digital radiographs. $J$ Clin Orthop Trauma. 2015;6(4):215-9.

12. Petretta R, Strelzow J, Ohly NE, et al. Acetate templating on digital images is more accurate than computer-based templating for total hip arthroplasty. Clin Orthop Relat Res. 2015;473(12):3752-9.

13. Brew CJ, Simpson PM, Whitehouse SL, et al. Scaling digital radiographs for templating in total hip arthroplasty using conventional acetate templates independent of calibration markers. $J$ Arthroplasty. 2012;27(4):643-7.

14. Conn KS, Clarke MT, Hallett JP. A simple guide to determine the magnification of radiographs and to improve the accuracy of preoperative templating. J Bone Joint Surg Br. 2002;84(2):269-72.

15. Boese CK, Lechler P, Rose L, et al. Calibration markers for digital templating in total hip arthroplasty. PLoS ONE. 2015;10(7):e0128529.

16. Viera AJ, Garrett JM. Understanding interobserver agreement: the kappa statistic. Fam Med. 2005;37(5):360-3.

17. Riddick A, Smith A, Thomas DP. Accuracy of preoperative templating in total hip arthroplasty. J Orthop Surg (Hong Kong). 2014;22(2):1736.

18. Clarke IC, Gruen T, Matos M, et al. Improved methods for quantitative radiographic evaluation with particular reference to total-hip arthroplasty. Clin Orthop Relat Res. 1976;(121):83-91.

19. Sinclair VF, Wilson J, Jain NPM, et al. Assessment of accuracy of marker ball placement in pre-operative templating for total hip arthroplasty. J Arthroplasty. 2014;29(8):1658-60.

20. The B, Diercks RL, van Ooijen PMA, et al. Comparison of analog and digital preoperative planning in total hip and knee arthroplasties. A prospective study of 173 hips and 65 total knees. Acta Orthop. 2005;76(1):78-84.

21. Bayne CO, Krosin M, Barber TC. Evaluation of the accuracy and use of X-Ray markers in digital templating for total hip Arthroplasty. $J$ Arthroplasty. 2009;24(3):407-13.

22. Franken M, Grimm B, Heyligers I. A comparison of four systems for calibration when templating for total hip replacement with digital radiography. J Bone Joint Surg Br. 2010;92(1):136-41.

23. Heinert G, Hendricks J, Loeffler MD. Digital templating in hip replacement with and without radiological markers. J Bone Joint Surg Br. 2009;91(4):459-62.

24. Descamps S, Livesey C, Learmonth ID. Determination of digitised radiograph magnification factors for pre-operative templating in hip prosthesis surgery. Skeletal Radiol. 2010;39(3):273-7.

25. Sershon RA, Diaz A, Bohl DD, et al. Effect of body mass index on digital templating for total hip arthroplasty. J Arthroplasty. 2017;32(3):10241026.

26. Kniesel B, Konstantinidis L, Hirschmüller A, et al. Digital templating in total knee and hip replacement: an analysis of planning accuracy. Int Orthop. 2014;38(4):733-9.

27. Rimm EB, Stampfer MJ, Colditz GA, et al. Validity of self-reported waist and hip circumferences in men and women. Epidemiology. 1990;1(6):466-73. 